\title{
Remark on: "Exp-function method for the exact solutions of fifth order KdV equation and modified Burgers equation" [Appl. Math. Comput. (2009) doi:10.1016/j.amc.2009.07.009]
}

\author{
İsmail Aslan \\ Department of Mathematics, Izmir Institute of Technology, Urla, Izmir 35430, Turkey
}

\section{A R T I C L E I N F O}

\section{Keywords:}

Exp-function method

Fifth order KdV equation

Modified Burgers equation

Common error

\begin{abstract}
A B S T R A C T
By means of the Exp-function method, Inan and Ugurlu [Appl. Math. Comput. (2009) doi:10.1016/j.amc.2009.07.009] reported eight expressions for being solutions to the two equations studied. In fact, all of them can be easily simplified to constants.
\end{abstract}

(c) 2010 Elsevier Inc. All rights reserved.

As a first example, Inan and Ugurlu [1] considered the fifth order KdV Eq. (5). According to the Exp-function method [2], they took the ansatz (8) into account and derived the solution sets (10)-(14) of the resultant algebraic system. However, we observed that the solution sets (11)-(14) are already included in the solution set (10), so they are redundant. Corresponding to (10), they obtained the solution (15) which can be easily reduced to the constant $-2 w / k$.

As a second example, they studied the modified Burgers Eq. (20). By means of the ansatz (23), they obtained the solution sets (25)-(27) of the resultant algebraic system. But, we observed that the solution set (25) is already included in the solution set (27), so it is redundant. Corresponding to (26) and (27), they derived the solutions (29) and (30) which can be easily reduced to the constants $\sqrt{3 k}$ and $\sqrt{3 k / 2}$, respectively.

The Exp-function method is entirely algorithmic and almost impossible to handle without a computer. It is based on trying rational combinations of exponential functions, as an ansatz, involving many unknown parameters to be specified at the stage of solving the problem. In the research literature, there have been some precedents for "solutions" revealed by the Exp-function method might be misleading, see [3-7]. Thus, one must be eagle-eyed to solve an equation by the Exp-function method. The equations considered in [1] already assume constant solutions since each term of the equations contains a derivative. It is unfortunate that the authors added one more example to the list of poor applications of the Exp-function method.

\section{References}

[1] I.E. Inan, Y. Ugurlu, Exp-function method for the exact solutions of fifth order KdV equation and modified Burgers equation, Appl. Math. Comput. 217 (2010) 1294-1299.

[2] J.H. He, X.H. Wu, Exp-function method for nonlinear wave equations, Chaos Soliton. Fract. 30 (2006) 700-708.

[3] N.A. Kudryashov, Seven common errors in finding exact solutions of nonlinear differential equations, Commun. Nonlinear Sci. Numer. Simulat. 14 (2009) 3507-3529.

[4] N.A. Kudryashov, N.B. Loguinova, Be careful with the Exp-function method, Commun. Nonlinear Sci. Numer. Simulat. 14 (2009) $1881-1890$.

[5] I. Aslan, Comment on: "New exact solutions for the Kawahara equation using Exp-function method" [J. Comput. Appl. Math. 233 (2009) 97-102], J. Comput. Appl. Math. 234 (2010) 3213-3215.

[6] N.A. Kudryashov, Comment on: "A novel approach for solving the Fisher equation using Exp-function method" [Phys. Lett. A 372 (2008) 3836], Phys. Lett. A 373 (2009) 1196-1197.

[7] N.A. Kudryashov, A note on new exact solutions for the Kawahara equation using Exp-function method, J. Comput. Appl. Math. 234 (2010) $3511-3512$.

E-mail address: ismailaslan@iyte.edu.tr 